J. Korean Math. Soc. 50 (2013), No. 1, pp. 1-16

http://dx.doi.org/10.4134/JKMS.2013.50.1.001

\title{
REPRESENTATION THEOREMS FOR MULTIVALUED PRAMARTS
}

\author{
Fettah Akhiat and Fatima Ezzaki
}

\begin{abstract}
Existence of pramarts selectors for multivalued pramart whose values are convex weakly compact subsets of a separable Banach space $E$ (resp. subsets of a dual space $E^{*}$ ) are established. Representation theorems for multivalued pramarts are also presented.
\end{abstract}

\section{Introduction}

Representation theorems of multivalued martingales, submartingales, supermartingales and uniform-amarts have been extensively studied in recent years by A. Choukairi [7], C. Hess [11], D. Q. Luu [14], Z. P. Wang and X. H. Xue [19] and $\mathrm{S}$. Li and Y. Ogura [13]. It is known that every multivalued martingale is a multivalued submartingale and is a supermartingale and also a uniform-amart. So, any uniform-amart is a pramart. A naturel questions raised by A. Choukairi in [8] is the existence of pramarts selectors for multivalued pramart. The main purpose of this work is not only to solve this problem but also prove that a multivalued pramart has a Castaing representation by pramarts selectors. The paper is organized as follows. In Section 2 we recall some notations and definitions and summarize needed results. In Section 3 we give some decomposition results for convex weakly compact valued pramarts. In Section 4 we discuss the existence of pramart selectors of convex weakly compact valued pramarts. In Section 5 we present a decomposition results for multivalued pramart whose values are convex weakly compact in the dual of a separable Banach space and we show the existence of pramarts selectors of the above class of pramarts.

\section{Preliminaries and background}

Throughout this paper $(\Omega, \mathcal{F}, P)$ is a complete probability space, $\left(\mathcal{F}_{n}\right)_{n \geq 1}$ an increasing sequence of sub $\sigma$-algebras of $\mathcal{F}$ such that $\mathcal{F}$ is the $\sigma$-algebra generated by $\cup_{n \geq 1} \mathcal{F}_{n} . E$ is a separable Banach space with the dual $E^{*}$ and the strong dual $E_{b}^{*}$. $\bar{B}_{E}$ (resp. $\left.\bar{B}_{E^{*}}\right)$ the closed unit ball of $E$ (resp. $E^{*}$ ).

Received May 11, 2011; Revised May 21, 2012.

2010 Mathematics Subject Classification. Primary 28B20, 60G42, 46A17, 46A20, 54A20.

Key words and phrases. multifunctions, Banach space, dual space, pramarts, subpramarts, pramarts selectors. 
$2^{E}$ is the set of all subsets of $E$. Let $c c(E)$ (resp. $c w k(E)$ ) be the set of nonempty convex closed subsets of $E$ (resp. weakly compact subsets of $E$ ). For $A \in 2^{E} \backslash \emptyset$, we denote by $\operatorname{cl} A$ and $\overline{c o} A$ the closure and the closed convex hull of $A$ respectively, and define $|A|=\sup \{\|x\|: x \in A\}$, the distance function and the support function associated with $A$ are defined respectively by

$$
\begin{gathered}
d(x, A)=\inf \{\|x-y\|, y \in A\} \quad(x \in E) . \\
\delta^{*}\left(x^{*}, A\right)=\sup \left\{\left\langle x^{*}, y\right\rangle, y \in A\right\}\left(x^{*} \in E^{*}\right) .
\end{gathered}
$$

The Hausdorff distance between $A$ and $B$ is denoted by

$$
\mathcal{H}(A, B)=\sup _{x^{*} \in \bar{B}_{E^{*}}}\left|\delta^{*}\left(x^{*}, A\right)-\delta^{*}\left(x^{*}, B\right)\right| .
$$

The equivalent definition of Hausdorff distance is

$$
\mathcal{H}(A, B)=\max \{\inf \{\lambda: B \subset A+\lambda\}, \inf \{\lambda: A \subset B+\lambda\}\},
$$

where

$$
A+\lambda=\{x: d(x, A) \leq \lambda\} .
$$

A multifunction (mappings for short) $X$ is a map from $\Omega$ into $2^{E}$. The domain of $X$ is defined by

$$
\operatorname{dom}(X)=\{\omega \in \Omega: X(\omega) \neq \emptyset\} .
$$

A selector of $X$ is a function $f: \Omega \longrightarrow E$ such that $f(\omega)$ is a member of $X(\omega)$ for all $\omega \in \operatorname{dom}(X)$.

A multifunction $X: \Omega \longrightarrow 2^{E}$ is said to be measurable, if for every open set $U \subset E$, the set

$$
X^{-} U=\{\omega \in \Omega: X(\omega) \cap U \neq \emptyset\}
$$

is a member of $\mathcal{F}$ (see [6], [12]). A measurable multifunction is also called a random set. For each $n \in \mathbb{N} \cup\{\infty\}$, we denote by $\mathcal{L}_{c w k(E)}^{1}\left(\mathcal{F}_{n}\right)\left(\operatorname{resp} . \mathcal{L}_{c c(E)}^{1}\left(\mathcal{F}_{n}\right)\right)$ the space of all $\mathcal{F}_{n}$-measurable $c w k(E)$-valued multifunctions $X: \Omega \rightarrow c w k(E)$ (resp. $c c(E)$-valued multifunctions $X: \Omega \rightarrow c c(E)$ ) such that $\omega \rightarrow|X(\omega)|$ is integrable. A sequence $\left(X_{n}\right)_{n \in \mathbb{N}}$ of $c c(E)$-valued multifunctions is adapted if each $X_{n}$ is $\mathcal{F}_{n}$-measurable. A measurable selector of the random set $X$ is an $(\mathcal{F}, \mathcal{B}(E))$-measurable selector of $X$. A Castaing representation [6] of $X$ is a sequence $f_{n}: \Omega \longrightarrow E$ of measurable selectors of $X$ such that

$$
X(\omega)=\operatorname{cl}\left\{f_{k}(\omega): k \geq 1\right\} \quad \text { for all } \omega \in \operatorname{dom}(X) .
$$

We denote by $L_{E}^{1}(\mathcal{F})$ the space of (equivalence classes of $)(\mathcal{F}, \mathcal{B}(E))$-measurable functions $f: \Omega \longrightarrow E$ such that $\omega \longrightarrow\|f(\omega)\|$ is integrable. Such an $f$ is said to be Bochner integrable. For every multifunction $X: \Omega \longrightarrow 2^{E}$ and every sub- $\sigma$-algebra $\mathcal{B}$ of $\mathcal{F}$, we set

$$
S_{X}^{1}(\mathcal{B})=\left\{f \in L_{E}^{1}(\mathcal{B}): f(\omega) \in X(\omega) \text { a.s. }\right\} .
$$

It is known that $S_{X}^{1}(\mathcal{B})$ characterizes $X$ up to $P$-null sets (see [12]). A measurable multifunction $X$ such that $S_{X}^{1}(\mathcal{B})$ is nonempty is declared integrable. Using Hiai and Umegaki [12, Theorem 2.2], it is readily seen that $X$ is integrable 
if and only if $d(0, X(\cdot)) \in L_{E}^{1}$. Now, consider an integrable $\mathcal{F}$-measurable multifunction $X: \Omega \longrightarrow c c(E)$. Following Hiai and Umegaki we define the multivalued conditional expectation of $X$ relative to $\mathcal{B}$ as the $\mathcal{B}$-measurable random set $G=E^{\mathcal{B}} X$ such that $S_{G}^{1}(\mathcal{B})=\operatorname{cl}\left\{E^{\mathcal{B}} f: f \in S_{X}^{1}(\mathcal{F})\right\}$, the closure being taken in $L_{E}^{1}$ (where $E^{\mathcal{B}} f$ denotes the usual conditional expectation relative to $\mathcal{B}$ of a Bochner integrable function $f$ ). In the special case where $\mathcal{B}=\{E, \emptyset\}$, $E^{\mathcal{B}} X$ is simply denoted by $E(X)$ and is equal to $c l\left\{E(f): f \in S_{X}^{1}(\mathcal{F})\right\}$. New existence results of conditional expectation for convex weakly compact valued multifunctions and its applications to martingales are available in $[1,3]$.

We denote by $\mathbb{T}$ the set of all bounded stopping times. A sequence $\left(X_{n}\right)_{n \geq 1}$ in $L_{E}^{1}(\mathcal{F})$ is of class $(B)$ if

$$
\sup _{\tau \in \mathbb{T}} \int_{\Omega}\left\|X_{\tau}\right\| d P<\infty
$$

\section{Decomposition theorems for multivalued pramarts}

Before going further, let us introduce the definitions of pramart in $\mathcal{L}_{c w k(E)}^{1}(\mathcal{F})$ and $L_{E}^{1}(\mathcal{F})$.

Definition 3.1. An adapted sequence $\left(X_{n}, \mathcal{F}_{n}\right)_{n \in \mathbb{N}}$ in $L_{E}^{1}(\mathcal{F})$ is a pramart if, for every $\varepsilon>0$, there is $\sigma_{\varepsilon} \in \mathbb{T}$ such that

$$
\forall \sigma, \tau \in \mathbb{T}, \quad \tau \geq \sigma \geq \sigma_{\varepsilon} \Rightarrow P\left(\left[\left\|X_{\sigma}-E^{\mathcal{F}_{\sigma}} X_{\tau}\right\|>\varepsilon\right]\right)<\varepsilon .
$$

Definition 3.2. An adapted sequence $\left(X_{n}, \mathcal{F}_{n}\right)_{n \in \mathbb{N}}$ in $\mathcal{L}_{c w k(E)}^{1}(\mathcal{F})$ is a pramart if, for every $\varepsilon>0$, there is $\sigma_{\varepsilon} \in \mathbb{T}$ such that

$$
\forall \sigma, \tau \in \mathbb{T}, \quad \tau \geq \sigma \geq \sigma_{\varepsilon} \Rightarrow P\left(\left[\mathcal{H}\left(X_{\sigma}, E^{\mathcal{F}_{\sigma}} X_{\tau}\right)>\varepsilon\right]\right)<\varepsilon .
$$

It is clear that if $\left(X_{n}, \mathcal{F}_{n}\right)_{n \in \mathbb{N}}$ is a pramart in $\mathcal{L}_{c w k(E)}^{1}(\mathcal{F})$, then, for each $x^{*} \in \bar{B}_{E^{*}}$, the adapted sequence $\left(\delta^{*}\left(x^{*}, X_{n}\right), \mathcal{F}_{n}\right)_{n \in \mathbb{N}}$ is a real-valued pramart in $L_{\mathbb{R}}^{1}(\mathcal{F})$ because

$$
\left|\delta^{*}\left(x^{*}, X_{\sigma}\right)-E^{\mathcal{F}_{\sigma}} \delta^{*}\left(x^{*}, X_{\tau}\right)\right| \leq \mathcal{H}\left(X_{\sigma}, E^{\mathcal{F}_{\sigma}} X_{\tau}\right) .
$$

Definition 3.3. An adapted sequence $\left(X_{n}, \mathcal{F}_{n}\right)_{n \in \mathbb{N}}$ in $L_{\mathbb{R}}^{1}(\mathcal{F})$ is a subpramart, if, for every $\varepsilon>0$, there is $\sigma_{\varepsilon} \in \mathbb{T}$ such that

$$
\forall \sigma, \tau \in \mathbb{T}, \quad \tau \geq \sigma \geq \sigma_{\varepsilon} \Rightarrow P\left(\left\{\left(X_{\sigma}-E^{\mathcal{F}_{\sigma}} X_{\tau}\right)^{+} \geq \varepsilon\right\}\right) \leq \varepsilon .
$$

Definition 3.4. Let $\left(X_{n}^{m}, \mathcal{F}_{n}\right)_{n \in \mathbb{N}}$ be a sequence of real subpramarts. It is called a uniform sequence of positive subpramarts if for every $\varepsilon>0$, there is $\sigma_{0} \in \mathbb{T}$ such that if $\sigma, \tau \in \mathbb{T}$ with $\tau \geq \sigma \geq \sigma_{0}$, then

$$
P\left(\left\{\sup _{m \in \mathbb{N}}\left(X_{\sigma}^{m}-E^{\mathcal{F}_{\sigma}} X_{\tau}^{m}\right)^{+} \geq \varepsilon\right\}\right) \leq \varepsilon .
$$

Now we proceed to the decomposition of $c w k(E)$-valued pramarts. 
Theorem 3.5. Assume that $E_{b}^{*}$ is separable. Let $\left(X_{n}, \mathcal{F}_{n}\right)_{n \geq 1}$ be a bounded pramart in $\mathcal{L}_{c w k(E)}^{1}(\mathcal{F})$ such that there exists a cwk $(E)$-valued multifunction $K: \Omega \Longrightarrow \operatorname{cwk}(E)$ satisfying $X_{n}(\omega) \subset K(w) \forall n \in \mathbb{N}, \forall \omega \in \Omega$. Then there exists a multifunction $X_{\infty} \in \mathcal{L}_{c w k(E)}^{1}(\mathcal{F})$ such that:

$$
\lim _{n \rightarrow \infty} \mathcal{H}\left(X_{n}, E^{\mathcal{F}_{n}} X_{\infty}\right)=0 \quad \text { a.s. }
$$

Proof. Step 1 Claim: $\lim _{n \rightarrow \infty} \delta^{*}\left(x^{*}, X_{n}\right)=\delta^{*}\left(x^{*}, X_{\infty}\right)$ a.s. $\forall x^{*} \in \bar{B}_{E^{*}}$.

Let $M_{1}^{*}=\left(f_{j}^{*}\right)_{j \in \mathbb{N}}$ be a dense sequence in $\bar{B}_{E^{*}}$ with respect to the Mackey topology $\tau\left(E^{*}, E\right)$. Since $\left(X_{n}\right)_{n \in \mathbb{N}}$ is bounded in $\mathcal{L}_{c w k(E)}^{1}(\mathcal{F})$, that is,

$$
\sup _{n \in \mathbb{N}} \int_{\Omega}\left|X_{n}\right| d P=\sup _{n \in \mathbb{N}} \int_{\Omega} \sup _{x^{*} \in \bar{B}_{E^{*}}}\left|\delta^{*}\left(x^{*}, X_{n}\right)\right| d P<\infty
$$

for each $j \in \mathbb{N}$, the $L^{1}$-bounded pramart $\left(\delta^{*}\left(f_{j}^{*}, X_{n}\right)\right)_{n \in \mathbb{N}}$ converge a.s. to an integrable function $\varphi_{x^{*}} \in L_{\mathbb{R}}^{1}(\mathcal{F})$. Let $\omega \in \Omega$, define the function $s(\cdot)$ by

$$
s\left(f_{j}^{*}\right)=\lim _{n \rightarrow \infty} \delta^{*}\left(f_{j}^{*}, X_{n}(\omega)\right) \quad(j \in \mathbb{N}) .
$$

$s$ is sublinear and continuous for the Mackey topology $\tau\left(E^{*}, E\right)$. Consequently, there is $X_{\infty}(\omega) \in \operatorname{cwk}(E)$ with $X_{\infty}(\omega) \subset K(\omega)$ such that

$$
s\left(f_{j}^{*}\right)=\delta^{*}\left(f_{j}^{*}, X_{\infty}(\omega)\right) \quad(j \in \mathbb{N}) .
$$

Then there exists a negligible set $N \in \mathcal{F}$ such that for all $\omega \in \Omega \backslash N$

$$
\lim _{n \rightarrow \infty} \delta^{*}\left(f_{j}^{*}, X_{n}(\omega)\right)=\delta^{*}\left(f_{j}^{*}, X_{\infty}(\omega)\right) \quad \forall j \in \mathbb{N} .
$$

Since the functions $\delta^{*}\left(\cdot, X_{n}(\omega)\right)$ and $\delta^{*}(\cdot, X(\omega))$ are continuous for the Mackey topology $\tau\left(E^{*}, E\right)$. So, we deduce that

$$
\lim _{n \rightarrow \infty} \delta^{*}\left(x^{*}, X_{n}\right)=\delta^{*}\left(x^{*}, X_{\infty}\right) \quad \text { a.s. } \quad \forall x^{*} \in \bar{B}_{E^{*}} .
$$

We check that $X_{\infty} \in \mathcal{L}_{c w k(E)}^{1}(\mathcal{F})$. Indeed, $X_{\infty}$ is measurable and for fixed $x^{*} \in \bar{B}_{E^{*}}$ and $\omega \in \Omega$, the function $n \longrightarrow \delta^{*}\left(x^{*}, X_{n}(\omega)\right)$ is continuous from $\mathbb{N} \cup\{+\infty\}$ into $\mathbb{R}$, therefore the function $n \rightarrow \sup \left\{\delta^{*}\left(x^{*}, X_{n}(\omega)\right): x^{*} \in \bar{B}_{E^{*}}\right\}$ is lower semi continuous on $\mathbb{N} \cup\{+\infty\}$ and so

$$
\left|X_{\infty}\right|(\omega) \leq \liminf _{n}\left|X_{n}\right|(\omega),
$$

by Fatou Lemma we have

$$
\begin{aligned}
\int_{\Omega}\left|X_{\infty}\right| d P & \leq \int_{\Omega} \liminf _{n}\left|X_{n}\right| d P \\
& \leq \liminf _{n} \int_{\Omega}\left|X_{n}\right| d P \leq \sup _{n} \int_{\Omega}\left|X_{n}\right| d P<+\infty
\end{aligned}
$$

Step 2 Claim: $\lim _{n \rightarrow \infty} \mathcal{H}\left(X_{n}, E^{\mathcal{F}_{n}} X_{\infty}\right)=0$ a.s.

Let $D_{1}^{*}=\left(e_{j}^{*}\right)_{j \in \mathbb{N}}$ be a dense sequence in the closed unit ball $\bar{B}_{E^{*}}$. We have

$$
\mathcal{H}\left(X_{n}, E^{\mathcal{F}_{n}} X_{\infty}\right)=\sup _{j \in \mathbb{N}}\left|\delta^{*}\left(e_{j}^{*}, X_{n}\right)-\delta^{*}\left(e_{j}^{*}, E^{\mathcal{F}_{n}} X_{\infty}\right)\right| .
$$


As $\left(\delta^{*}\left(e_{j}^{*}, X_{n}\right)-\delta^{*}\left(e_{j}^{*}, E^{\mathcal{F}_{n}} X_{\infty}\right)\right)_{n \in \mathbb{N}}$ are real-valued pramarts in $L_{\mathbb{R}}^{1}(\mathcal{F})$ which converges a.s. to 0 , and $\left(\left(\left|\delta^{*}\left(e_{j}^{*}, X_{n}\right)-\delta^{*}\left(e_{j}^{*}, E^{\mathcal{F}_{n}} X_{\infty}\right)\right|\right)_{n \in \mathbb{N}}\right)_{j \in \mathbb{N}}$ is a uniform sequence of positive subpramarts, applying lemma VIII.1.15 in [9] we have

$$
\begin{aligned}
\lim _{n \rightarrow \infty} \mathcal{H}\left(X_{n}, E^{\mathcal{F}_{n}} X_{\infty}\right) & =\lim _{n \rightarrow \infty} \sup _{j \in \mathbb{N}}\left|\delta^{*}\left(e_{j}^{*}, X_{n}\right)-\delta^{*}\left(e_{j}^{*}, E^{\mathcal{F}_{n}} X_{\infty}\right)\right| \\
& =\sup _{j \in \mathbb{N}} \lim _{n \rightarrow \infty}\left|\delta^{*}\left(e_{j}^{*}, X_{n}\right)-\delta^{*}\left(e_{j}^{*}, E^{\mathcal{F}_{n}} X_{\infty}\right)\right|=0
\end{aligned}
$$

almost surely.

Now we give a quasi-decomposition theorem for convex weakly compact valued pramart.

Theorem 3.6. Assume that $E_{b}^{*}$ is separable. Let $\left(X_{n}, \mathcal{F}_{n}\right)_{n \geq 1}$ be a bounded pramart in $\mathcal{L}_{c w k(E)}^{1}(\mathcal{F})$ such that, there exists a cwk $(E)$-valued multifunction $K: \Omega \Longrightarrow \operatorname{cwk}(E)$ satisfying $X_{n}(\omega) \subset K(w) \forall n \in \mathbb{N}, \forall \omega \in \Omega$. Then there exist a multivalued martingale $\left(M_{n}\right)_{n \geq 1}$ and $\left(Z_{n}\right)_{n \geq 1}$ in $\mathcal{L}_{c w k(E)}^{1}(\mathcal{F})$ such that

$$
\begin{aligned}
& X_{n}(w) \subset M_{n}(w)+Z_{n}(w) \quad \text { a.s. } \\
& \left|Z_{n}\right| \longrightarrow 0 \quad \text { a.s. as } n \rightarrow+\infty .
\end{aligned}
$$

Proof. By Theorem 3.5 there exists $X_{\infty} \in \mathcal{L}_{c w k(E)}^{1}(\mathcal{F})$ such that

$$
\lim _{n \rightarrow \infty} \mathcal{H}\left(X_{n}, E^{\mathcal{F}_{n}} X_{\infty}\right)=0 \quad \text { a.s. }
$$

Let $M_{n}=E^{\mathcal{F}_{n}} X_{\infty}$, then $\left(M_{n}\right)_{n \in \mathbb{N}}$ is a multivalued martingale, and

$$
\lim _{n \rightarrow \infty} \mathcal{H}\left(X_{n}, M_{n}\right)=0 \text { a.s. }
$$

If we set $\rho_{n}=\mathcal{H}\left(X_{n}, M_{n}\right)$, define $Z_{n}$ by

$$
Z_{n}=\left\{x \in E \backslash\|x\| \leq \mathcal{H}\left(X_{n}, M_{n}\right)=\rho_{n}\right\}=\bar{B}_{E}\left(0, \rho_{n}\right) .
$$

Then by definition of the Hausdorff distance we have

$$
X_{n}(w) \subset M_{n}(w)+\mathcal{H}\left(X_{n}(w), M_{n}(w)\right)=M_{n}(w)+\rho_{n}(w) \text { a.s. }
$$

So $X_{n}(w) \subset M_{n}(w)+Z_{n}(w)$ a.s. Indeed, we must prove that

$$
\left\{x: d\left(x, M_{n}(w)\right) \leq \rho_{n}(w)\right\}=M_{n}(w)+\bar{B}_{E}\left(0, \rho_{n}(w)\right) .
$$

First, if $d\left(x, M_{n}(w)\right) \leq \rho_{n}(w)$, then for each $k>0$, there exists $a_{k} \in M_{n}(w)$ such that

That is

$$
\left\|x-a_{k}\right\| \leq \rho_{n}(w)+\frac{1}{k}
$$

$$
x-a_{k} \in \bar{B}_{E}\left(0, \rho_{n}(w)+\frac{1}{k}\right) .
$$

So, there exists $y_{k} \in \bar{B}_{E}\left(0, \rho_{n}(w)+\frac{1}{k}\right)$ such that $x-a_{k}=y_{k}$ and

$$
x=a_{k}+y_{k} \in M_{n}(w)+\bar{B}_{E}\left(0, \rho_{n}(w)+\frac{1}{k}\right), \quad \forall k>0 .
$$


Since $a_{k} \in M_{n}(w)$ and $y_{k} \in \bar{B}_{E}\left(0, \rho_{n}(w)+\frac{1}{k}\right)$ this implies that there exists $a \in M_{n}(w)$ such that

$$
\lim _{k \rightarrow \infty}\left\langle x^{*}, a_{k}\right\rangle=\left\langle x^{*}, a\right\rangle \quad \forall x^{*} \in E^{*},
$$

and

$$
\begin{aligned}
\lim _{k \rightarrow \infty}\left\langle x^{*}, x-a_{k}\right\rangle & =\left\langle x^{*}, x-a\right\rangle \\
& =\lim _{k \rightarrow \infty}\left\langle x^{*}, y_{k}\right\rangle \\
& \leq \limsup _{k \rightarrow \infty} \delta^{*}\left(x^{*}, \bar{B}_{E}\left(0, \rho_{n}(w)+\frac{1}{k}\right)\right) .
\end{aligned}
$$

That is

$$
\left\langle x^{*}, x-a\right\rangle \leq \delta^{*}\left(x^{*}, \bar{B}_{E}\left(0, \rho_{n}(w)\right)\right) \quad \forall x^{*} \in E^{*} .
$$

According to Proposition III.35 in [6], we deduce that $y=x-a \in \bar{B}_{E}\left(0, \rho_{n}(w)\right)$. Finally $x \in M_{n}(w)+\bar{B}_{E}\left(0, \rho_{n}(w)\right)$.

Conversely, if $x \in M_{n}(w)+\bar{B}_{E}\left(0, \rho_{n}(w)\right)$, this implies that $\exists a \in M_{n}(w)$, $\exists z \in \bar{B}_{E}\left(0, \rho_{n}(w)\right)$ such that

$$
x=a+z .
$$

Indeed, since $x \in M_{n}(w)+\bar{B}_{E}\left(0, \rho_{n}(w)\right)$, then there exists $\left(x_{k}\right)_{k \geq 1}$ such that $x=\lim _{k} x_{k}$ and $x_{k}=a_{k}+z_{k}$ with $a_{k} \in M_{n}$ and $z_{k} \in \bar{B}_{E}\left(0, \rho_{n}(w)\right)$, hence there exist $k_{j}$ subsequence of $k$ and $a \in M_{n}$ such that

$$
\lim _{j \rightarrow \infty}\left\langle x^{*}, a_{k_{j}}\right\rangle=\left\langle x^{*}, a\right\rangle \text {. }
$$

On the other hand $x_{k_{j}}=a_{k_{j}}+z_{k_{j}}$ thus

$$
\lim _{j \rightarrow \infty}\left\langle x^{*}, x_{k_{j}}-a_{k_{j}}\right\rangle=\left\langle x^{*}, x-a\right\rangle=\lim _{j \rightarrow \infty}\left\langle x^{*}, z_{k_{j}}\right\rangle=\left\langle x^{*}, z\right\rangle .
$$

So, $z \in \bar{B}_{E}\left(0, \rho_{n}(w)\right)$ and $x=a+z$. Consequently

$$
\|x-a\|=\|z\| \leq \rho_{n}(w)
$$

and

$$
d\left(x, M_{n}(w)\right) \leq \rho_{n}(w) .
$$

Finally, $X_{n}(w) \subset M_{n}(w)+Z_{n}(w)$ a.s. and

$$
\left|Z_{n}\right| \leq \mathcal{H}\left(X_{n}, M_{n}\right) \longrightarrow_{n \rightarrow+\infty} 0 \text { a.s. }
$$

The following result is a consequence of Theorem 3.5.

Corollary 3.7. Assume that $E_{b}^{*}$ is separable. Let $\left(X_{n}, \mathcal{F}_{n}\right)_{n \geq 1}$ be a bounded pramart in $L_{E}^{1}(\mathcal{F})$ such that there exists a multifunction $K \in \mathcal{L}_{\text {cwk(E) }}^{1}(\mathcal{F})$ satisfying $X_{n}(\omega) \in K(w) \forall n \in \mathbb{N}, \forall \omega \in \Omega$. Then there are a unique regular martingale $\left(Y_{n}\right)$ in $L_{E}^{1}(\mathcal{F})$ and a pramart $\left(Z_{n}\right)$ in $L_{E}^{1}(\mathcal{F})$ such that

$$
X_{n}=Y_{n}+Z_{n}, \quad \forall n \in \mathbb{N},
$$

$$
\left|Z_{n}\right| \longrightarrow 0 \quad \text { a.s. as } n \rightarrow+\infty \text {. }
$$


Proof. By Theorem 3.5 there exists $X_{\infty}$ in $L_{E}^{1}(\mathcal{F})$ such that

$$
\lim _{n}\left\|X_{n}-E^{\mathcal{F}_{n}} X_{\infty}\right\|=0 \text { a.s. }
$$

Then, by setting $Y_{n}=E^{\mathcal{F}_{n}} X_{\infty}$ for all $n \in \mathbb{N}$, we have $X_{n}=Y_{n}+X_{n}-$ $Y_{n}=Y_{n}+Z_{n}$, where $Z_{n}=X_{n}-Y_{n}$ is obviously a pramart and $\lim _{n}\left\|Z_{n}\right\|=$ $\lim _{n}\left\|X_{n}-Y_{n}\right\|=0$ a.s. The uniqueness is more or less classical. Suppose

$$
X_{n}=Y_{n}^{\prime}+Z_{n}^{\prime}
$$

with the required properties in the corollary. Then

$$
\lim _{n \rightarrow \infty} Z_{n}=\lim _{n \rightarrow \infty} Z_{n}^{\prime}=0 \quad \text { a.s. }
$$

For each $m$ fixed in $\mathbb{N}$ we have

$$
\begin{gathered}
Y_{m}-Y_{m}^{\prime}=\lim _{n \rightarrow \infty}\left(E^{\mathcal{F}_{n}} Y_{m}-E^{\mathcal{F}_{n}} Y_{m}^{\prime}\right) \\
=\lim _{n \rightarrow \infty}\left(Y_{n}-Y_{n}^{\prime}\right)=\lim _{n \rightarrow \infty}\left(Z_{n}-Z_{n}^{\prime}\right)=0 \quad \text { a.s. }
\end{gathered}
$$

for every $m \in \mathbb{N}$.

\section{Representation theorems for multivalued pramarts}

In this present section we give our first results of existence of pramart selectors for $c w k(E)$-valued pramart.

Definition 4.1. A sequence $\left(f_{n}, \mathcal{F}_{n}\right)_{n \geq 1}$ is called a pramart selector of $\left(X_{n}, \mathcal{F}_{n}\right)_{n \geq 1}$ if

(i) $f_{n} \in S_{X_{n}}^{1}\left(\mathcal{F}_{n}\right)$ for all $n \in \mathbb{N}$.

(ii) $\left(f_{n}, \mathcal{F}_{n}\right)_{n \geq 1}$ is a pramart in $L_{E}^{1}(\mathcal{F})$. In this case we write $\left(f_{n}, \mathcal{F}_{n}\right)_{n \geq 1} \in$ $P S\left(X_{n}\right)$ and let $P S\left(X_{n}\right)$ denote the set of all pramart selectors of $\left(X_{n}, \mathcal{F}_{n}\right)_{n \geq 1}$.

To get further representation theorem, we need the following lemmas.

Lemma 4.2. Let $\left(X_{n}\right)_{n \geq 1}$ be a sequence in $L_{E}^{1}(\mathcal{F})$. If $\left(X_{n}\right)_{n \geq 1}$ is of class $(B)$ and $\left(X_{n}\right)$ converge in probability. Then $\left(X_{n}\right)_{n \geq 1}$ is a pramart in $L_{E}^{1}(\mathcal{F})$.

Proof. See [20, Lemma 6].

Lemma 4.3. If $\mathcal{B}_{1} \subset \mathcal{B}_{0}$ are two sub- $\sigma$-fields of $\mathcal{F}, X \in \mathcal{L}_{c c(E)}^{1}\left(\mathcal{B}_{1}\right), Y \in$ $\mathcal{L}_{c c(E)}^{1}\left(\mathcal{B}_{0}\right)$ and $\theta: \Omega \longrightarrow \mathbb{R}^{+} \backslash\{0\}$ is a $\mathcal{B}_{1}$-measurable function, then for each $f \in S_{X}^{1}\left(\mathcal{B}_{1}\right)$ we can find $g \in S_{Y}^{1}\left(\mathcal{B}_{0}\right)$ such that

$$
\left\|f(w)-E^{\mathcal{B}_{1}} g(w)\right\| \leq \mathcal{H}\left(X(w), E^{\mathcal{B}_{1}} Y(w)\right)+\theta(w) \text { a.s. }
$$

Consequently, if $Y$ is $\mathcal{B}_{1}$-measurable, then there exist some $g \in S_{Y}^{1}\left(\mathcal{B}_{1}\right)$ such that

$$
\|f(w)-g(w)\| \leq \mathcal{H}(X(w), Y(w))+\theta(w) \text { a.s. }
$$

Proof. See [16, Lemma 3.3]. 
Example 4.4. Let $\left(f_{n}, \mathcal{F}_{n}\right)_{n \geq 1}$ be a vector valued pramart and $\left(r_{n}, \mathcal{F}_{n}\right)_{n \geq 1}$ be a real valued pramart. Take $\bar{B}_{E}$ the closed unit ball of $E$, let $A \in \mathcal{F}$. Define

$$
X_{n}=f_{n} 1_{A}+r_{n} 1_{A^{c}} \bar{B}_{E} .
$$

Then $\left(X_{n}\right)_{n \geq 1}$ is a multivalued pramart. Indeed, for $\tau, \sigma \in \mathbb{T}(\tau \geq \sigma)$

$$
\begin{aligned}
& P\left(\mathcal{H}\left(X_{\sigma}, E^{\mathcal{F}_{\sigma}} X_{\tau}\right)>\varepsilon\right) \\
= & P\left(\mathcal{H}\left(f_{\sigma} 1_{A}+r_{\sigma} 1_{A^{c}} \bar{B}_{E}, E^{\mathcal{F}_{\sigma}}\left(f_{\tau} 1_{A}+r_{\tau} 1_{A^{c}} \bar{B}_{E}\right)\right)>\varepsilon\right) \\
\leq & P\left(\left\|f_{\sigma} 1_{A}-E^{\mathcal{F}_{\sigma}} f_{\tau} 1_{A}\right\|+\left|\bar{B}_{E}\right| \cdot\left|r_{\sigma} 1_{A^{c}}-E^{\mathcal{F}_{\sigma}} r_{\tau} 1_{A^{c}}\right|>\varepsilon\right) \\
\leq & P\left(\left\|f_{\sigma} 1_{A}-E^{\mathcal{F}_{\sigma}} f_{\tau} 1_{A}\right\|+\left|r_{\sigma} 1_{A^{c}}-E^{\mathcal{F}_{\sigma}} r_{\tau} 1_{A^{c}}\right|>\varepsilon\right) \\
\leq & P\left(\left\|f_{\sigma} 1_{A}-E^{\mathcal{F}_{\sigma}} f_{\tau} 1_{A}\right\|>\frac{\varepsilon}{2}\right)+P\left(\left|r_{\sigma} 1_{A^{c}}-E^{\mathcal{F}_{\sigma}} r_{\tau} 1_{A^{c}}\right|>\frac{\varepsilon}{2}\right) \\
\leq & \frac{\varepsilon}{2}+\frac{\varepsilon}{2}=\varepsilon .
\end{aligned}
$$

It is easy to see that every sequence $\left(g_{n}\right)_{n \geq 1}$ define by

$$
g_{n}=f_{n} 1_{A}+r_{n} 1_{A^{c}} x \quad \text { for each } x \in \bar{B}_{E}
$$

is a pramart selector of $\left(X_{n}\right)_{n \geq 1}$.

Definition 4.5. Given $\left(X_{n}\right)_{n \geq 1}$ in $\mathcal{L}_{c w k(E)}^{1}(\mathcal{F})$. We say that assumption $(A)$ holds, if every sequence of selectors of $\left(X_{n}\right)_{n \geq 1}$ is of class $(B)$.

Theorem 4.6. Assume that $E_{b}^{*}$ is separable. Let $\left(X_{n}, \mathcal{F}_{n}\right)_{n \geq 1}$ be a bounded pramart in $\mathcal{L}_{c w k(E)}^{1}(\mathcal{F})$ such that there exists a cwk $(E)$-valued multifunction $K: \Omega \Longrightarrow c w k(E)$ satisfying $X_{n}(\omega) \subset K(w) \forall n \in \mathbb{N}, \forall \omega \in \Omega$ and if assumption (A) holds. Then

$$
S_{X_{k}}^{1}\left(\mathcal{F}_{k}\right)=\pi_{k}\left(P S\left(X_{n}\right)\right) .
$$

Where for every $\left(f_{n}\right) \in P S\left(X_{n}\right), \pi_{k}\left(\left(f_{n}\right)\right)=f_{k}\left(\pi_{k}\right.$ is the usual projection to the $k$ th element of the sequence $\left.\left(f_{n}\right)_{n \geq 1}\right)$.

Proof. By Theorem 3.5 there is $X_{\infty} \in \mathcal{L}_{c w k(E)}^{1}(\mathcal{F})$ such that

$$
\lim _{n \rightarrow \infty} \mathcal{H}\left(X_{n}, E^{\mathcal{F}_{n}} X_{\infty}\right)=0 \quad \text { a.s. }
$$

Let $M_{n}=E^{\mathcal{F}_{n}} X_{\infty}$, for each $n \in \mathbb{N}$ we set $\rho_{n}(w)=\mathcal{H}\left(X_{n}(w), M_{n}(w)\right)$, and let $r_{n}(w)=\rho_{n}(w)+\frac{1}{2^{n}}, \forall n \geq 1$.

Now, let $k \geq 1$ and let $\widehat{f}_{k} \in S_{X_{k}}^{1}\left(\mathcal{F}_{k}\right)$. From [14] see also [11], we know that there exists a sequence $\left(h_{n}^{i}, \mathcal{F}_{n}\right)_{i \geq 1}$ in $M S\left(M_{n}\right)$ (here $M S\left(M_{n}\right)$ is the set of all martingales selectors of $\left.M_{n}\right)$ such that for every $n \geq 1$,

$$
M_{n}(w)=\operatorname{cl}\left\{h_{n}^{i}(\omega) ; \quad i \geq 1\right\}, \quad \forall \omega \in \Omega .
$$

Define $\tau: \Omega \longrightarrow \mathbb{N}$ and $h_{k}^{\tau}: \Omega \longrightarrow E$ by

$$
\tau(w)=\inf \left\{i \geq 1,\left\|\widehat{f}_{k}(\omega)-h_{k}^{i}(\omega)\right\| \leq d\left(\widehat{f}_{k}(w), M_{k}(w)\right)+\frac{1}{2^{k}}\right\}
$$


and

$$
h_{k}(w)=\sum_{i=1}^{+\infty} 1_{\{\tau=i\}}(\omega) h_{k}^{i}(w)=h_{k}^{\tau(\omega)}(w) .
$$

Obviously $\tau \in \mathcal{F}_{k}$ and $h_{k}(w) \in M_{k}(w)$. Also we have

$$
\begin{aligned}
\left\|\widehat{f}_{k}(w)-h_{k}(w)\right\| & =\sum_{i \geq 1} 1_{\{\tau=i\}}(\omega)\left\|\widehat{f}_{k}(w)-h_{k}^{i}(w)\right\| \\
& \leq d\left(\widehat{f}_{k}(w), M_{k}(w)\right)+\frac{1}{2^{k}} \leq r_{k}(w) \quad \text { a.s. }
\end{aligned}
$$

Next define

$$
h_{n}(w)= \begin{cases}\sum_{i \geq 1} 1_{\{\tau=i\}}(\omega) h_{n}^{i}(w), & \text { if } \quad n \geq k ; \\ E\left(h_{k}(w) / \mathcal{F}_{n}\right), & \text { if } \quad n<k .\end{cases}
$$

Then $\left(h_{n}, \mathcal{F}_{n}\right)_{n \geq 1}$ is in $M S\left(M_{n}\right)$. For each $h_{n}(w) \in M_{n}(w)$ by using Lemma 4.3 we can find a sequence $f_{n} \in S_{X_{n}}^{1}\left(\mathcal{F}_{n}\right)$ such that

$$
\left\|f_{n}(w)-h_{n}(w)\right\| \leq r_{n}(w) \quad \text { a.s. }
$$

Next we shall prove that $\left(f_{n}, \mathcal{F}_{n}\right)_{n \geq 1} \in P S\left(X_{n}\right)$. Indeed, firstly we can write $f_{n}=h_{n}+\left(f_{n}-h_{n}\right)=h_{n}+z_{n}$ where $z_{n}=f_{n}-h_{n}$, on the other hand from (4.6.2) $z_{n}$ converge to zero a.s. as $n \rightarrow+\infty$, and

$$
\int_{\Omega}\left\|z_{\tau}\right\| d P \leq \int_{\Omega}\left\|f_{\tau}\right\| d P+\int_{\Omega}\left|X_{\infty}\right| d P
$$

then

$$
\sup _{\tau \in \mathbb{T}} \int_{\Omega}\left\|z_{\tau}\right\| d P \leq \sup _{\tau \in \mathbb{T}} \int_{\Omega}\left\|f_{\tau}\right\| d P+\int_{\Omega}\left|X_{\infty}\right| d P<\infty .
$$

Hence by Lemma $4.2, z_{n}$ is a pramart. This with $\left(h_{n}\right)_{n \geq 1}$ being a martingale, implies that $\left(f_{n}\right)_{n \geq 1}$ is a pramart and it's martingale component in the decomposition of Corollary 3.7 is given by $\left(h_{n}\right)_{n \geq 1}$. By (4.6.1) and (4.6.2) we can take $f_{k}=\widehat{f}_{k}$ and so $\widehat{f}_{k} \in \pi_{k}\left(\left(f_{n}\right)\right) \in \pi_{k}\left(P S\left(X_{n}\right)\right)$. Hence $S_{X_{k}}^{1}\left(\mathcal{F}_{k}\right) \subset \pi_{k}\left(P S\left(X_{n}\right)\right)$. It is obvious that $S_{X_{k}}^{1}\left(\mathcal{F}_{k}\right) \supset \pi_{k}\left(P S\left(X_{n}\right)\right)$. So we have the result.

Now we are ready to state the following representation theorem of $c w k(E)$ valued pramarts.

Theorem 4.7. Assume that $E_{b}^{*}$ is separable. Let $\left(X_{n}\right)_{n \geq 1}$ be a pramart in $\mathcal{L}_{\text {cwk(E) }}^{1}(\mathcal{F})$ such that, there exists a cwk $(E)$-valued multifunction $K: \Omega \Longrightarrow$ cwk $(E)$ satisfying $X_{n}(\omega) \subset K(w) \quad \forall n \in \mathbb{N}, \forall \omega \in \Omega$ and if assumption $(A)$ holds. Then there exists a sequence $\left(f_{n}^{k}\right)_{k \geq 1}$ in $P S\left(X_{n}\right)$ such that for every $n \geq 1$,

$$
X_{n}(w)=c l\left\{f_{n}^{k}(w), k \geq 1\right\}, \quad \forall \omega \in \Omega .
$$


Proof. By Castaing representation theorem, we have that, for any $k \in \mathbb{N}$, there exists a sequence $\left\{g^{k, i}: i \in \mathbb{N}\right\} \subset S_{X_{k}}^{1}\left(\mathcal{F}_{k}\right)$ such that $X_{k}(w)=\operatorname{cl}\left\{g^{k, i}(w): i \in\right.$ $\mathbb{N}\}$ for all $\omega \in \Omega$. By virtue of Theorem 4.6, there exists a sequence of pramart selectors $\left\{h_{n}^{k, i, j}: j \in \mathbb{N}\right\}$ in $P S\left(X_{n}\right)$ such that

$$
\lim _{j \rightarrow \infty}\left\|\pi_{k}\left(h_{n}^{k, i, j}\right)-g^{k, i}\right\|_{1}=0 \quad \text { for all } k, i \in \mathbb{N} .
$$

Then

$$
\lim _{j \rightarrow \infty}\left\|h_{k}^{k, i, j}-g^{k, i}\right\|_{1}=0 \quad \text { for all } \quad k, i \in \mathbb{N} .
$$

But as from every $L^{1}$-convergent sequence we can extract an almost surely convergent subsequence, so by (4.7.1) without any loss of generality we have

$$
X_{k}(w)=\operatorname{cl}\left\{h_{k}^{k, i, j}(w): i, j \in \mathbb{N}\right\} \quad \text { for all } k \in \mathbb{N} .
$$

Finally, if $\left\{f_{n}^{l}: l \in \mathbb{N}\right\}$ denotes the sequence $\left\{h_{n}^{k, i, j}: k, i, j \in \mathbb{N}\right\}$, then the result is automatically satisfied.

\section{Multivalued pramarts in dual space}

Let $(\Omega, \mathcal{F}, P)$ be a complete probability space, $\left(\mathcal{F}_{n}\right)_{n \in \mathbb{N}}$ an increasing sequence of sub- $\sigma$-algebras of $\mathcal{F}$ such that $\mathcal{F}$ is the $\sigma$-algebra generated by $\cup_{n \geq 1} \mathcal{F}_{n}$. Let $E$ be a separable Banach space, $E^{*}$ the topological dual of $E$, $\bar{B}_{E}$ (resp. $\left.\bar{B}_{E^{*}}\right)$ the closed unit ball of $E$ (resp. $\left.E^{*}\right), D=\left(x_{p}\right)_{p \in \mathbb{N}}$ a dense sequence in $\bar{B}_{E}$. We denote by $E_{b}^{*}$ the strong dual endowed with the topology associated with the dual norm $\|\cdot\|_{E_{b}^{*}}$, by $E_{s}^{*}$ the topological dual $E^{*}$ endowed with the topology $\sigma\left(E^{*}, E\right)$ of pointwise convergence, alias $w^{*}$ topology. Noting that $E^{*}$ is the countable union of closed balls, we deduce that the space $E_{s}^{*}$

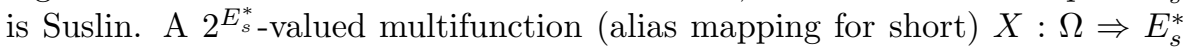
is $\mathcal{F}$-measurable if its graph belongs to $\mathcal{F} \otimes \mathcal{B}\left(E_{s}^{*}\right)$. Given a $\mathcal{F}$-measurable mapping $X: \Omega \Rightarrow E_{s}^{*}$ and a Borel set $G \in \mathcal{B}\left(E_{s}^{*}\right)$, the set

$$
X^{-} G=\{\omega \in \Omega: X(\omega) \cap G \neq \emptyset\}
$$

is $\mathcal{F}$-measurable, that is $X^{-} G \in \mathcal{F}$. In view of the completeness hypothesis on the probability space, this is a consequence of the Projection Theorem (see e.g. Theorem III.23 of [6]) and of the equality

$$
X^{-} G=\operatorname{proj}_{\Omega}\{G r(X) \cap(\Omega \times G)\} .
$$

In particular, if $X: \Omega \Rightarrow E_{s}^{*}$ is $\mathcal{F}$-measurable, the domain of $X$, defined by

$$
\operatorname{dom} X=\{\omega \in \Omega: X(\omega) \neq \emptyset\}
$$

is $\mathcal{F}$-measurable, because $\operatorname{dom} X=X^{-} E_{s}^{*}$. Here $L_{E^{*}}^{1}[E](\mathcal{F})$ is the space of all $\mathcal{F}$-measurable mappings $u: \Omega \longrightarrow E_{s}^{*}$ such that the function $|u|: \omega \mapsto$ $\|u(\omega)\|_{E_{b}^{*}}$ is integrable. For any $2^{E_{s}^{*}}$-valued mapping $X: \Omega \Rightarrow E_{s}^{*}$, we denote by $\mathcal{S}_{X}^{1}(\mathcal{F})$ the set of all $L_{E^{*}}^{1}[E](\mathcal{F})$-selectors of $X$. By $c w k\left(E_{s}^{*}\right)$ we denote the set of all nonempty convex $\sigma\left(E^{*}, E\right)$-compact subsets of $E_{s}^{*}$. A mapping $X: \Omega \rightarrow \operatorname{cwk}\left(E_{s}^{*}\right)$ is scalarly $\mathcal{F}$-measurable if the function $\omega \rightarrow$ 
$\delta^{*}(x, X(\omega))$ is $\mathcal{F}$-measurable for every $x \in E$. Let us recall that any scalarly $\mathcal{F}$-measurable $c w k\left(E_{s}^{*}\right)$-valued mapping is $\mathcal{F}$-measurable. Indeed, let $\left(e_{k}\right)_{k \in \mathbb{N}}$ be a sequence in $E$ which separates the points of $E^{*}$, then we have $x \in X(\omega)$ if and only if, $\left\langle e_{k}, x\right\rangle \leq \delta^{*}\left(e_{k}, X(\omega)\right)$ for all $k \in \mathbb{N}$. Further, we denote by $\mathcal{L}_{c w k\left(E_{s}^{*}\right)}^{1}(\Omega, \mathcal{F}, P)\left(\right.$ shortly $\left.\mathcal{L}_{c w k\left(E_{s}^{*}\right)}^{1}(\mathcal{F})\right)$ the space of all integrably bounded multifunctions $X$ such that the function $|X|: \omega \rightarrow|X(\omega)|$ is integrable, here $|X(\omega)|:=\left.\sup _{y^{*} \in X(\omega)}|| y^{*}\right|_{E_{b}^{*}}$, by the above consideration, it is easy to see that $|X|$ is $\mathcal{F}$-measurable. Let $\mathcal{H}_{E_{b}^{*}}^{*}$ be the Hausdorff distance associated with the dual norm $\|\cdot\|_{E_{b}^{*}}$ on bounded closed convex subsets in $E^{*}$, and $X, Y$ be two convex weak ${ }^{*}$ compact valued measurable mapping, then $\mathcal{H}_{E_{b}^{*}}^{*}(X, Y)$ is measurable because $\mathcal{H}_{E_{b}^{*}}^{*}(X, Y)=\sup _{j \in \mathbb{N}}\left[\delta^{*}\left(e_{j}, X\right)-\delta^{*}\left(e_{j}, Y\right)\right]$, where $\left(e_{j}\right)_{j \in \mathbb{N}}$ is a dense sequence in $\bar{B}_{E}$. A sequence $\left(X_{n}\right)_{n \in \mathbb{N}}$ in $\mathcal{L}_{c w k\left(E_{s}^{*}\right)}^{1}(\mathcal{F})$ is bounded if $\left(\left|X_{n}\right|\right)_{n \in \mathbb{N}}$ is bounded in $L_{\mathbb{R}}^{1}(\Omega, \mathcal{F}, P)$ (shortly $L_{\mathbb{R}}^{1}(\mathcal{F})$ ). For the existence and uniqueness of the conditional expectation in $\mathcal{L}_{c w k\left(E_{s}^{*}\right)}^{1}(\mathcal{F})$ and $L_{E^{*}}^{1}[E](\mathcal{F})$, we refer the reader to $[3,18]$. In the sequel we assume that $E_{b}^{*}$ is separable.

Before going further, let us introduce the definition of pramarts in $\mathcal{L}_{c w k\left(E_{s}^{*}\right)}^{1}(\mathcal{F})$ and $L_{E^{*}}^{1}[E](\mathcal{F})$.

Definition 5.1. An adapted sequence $\left(X_{n}\right)_{n \in \mathbb{N}}$ in $\mathcal{L}_{c w k\left(E_{s}^{*}\right)}^{1}(\mathcal{F})$ is a pramart if for every $\varepsilon>0$, there exists $\sigma_{\varepsilon} \in \mathbb{T}$ such that

$$
\forall \sigma, \tau \in \mathbb{T}, \quad \tau \geq \sigma \geq \sigma_{\varepsilon} \Rightarrow P\left(\mathcal{H}_{E_{b}^{*}}^{*}\left(X_{\sigma}, E^{\mathcal{F}_{\sigma}} X_{\tau}\right)>\varepsilon\right)<\varepsilon,
$$

where $\mathcal{H}_{E_{b}^{*}}^{*}$ stands for the Hausdorff distance associated with the dual norm $\|\cdot\|_{E_{b}^{*}}$ on $c w k\left(E_{s}^{*}\right)$.

Further, if $\left(X_{n}\right)_{n \in \mathbb{N}}$ is single-valued, definition 5.1 is reduced to:

Definition 5.2. An adapted sequence $\left(X_{n}\right)_{n \in \mathbb{N}}$ in $L_{E^{*}}^{1}[E](\mathcal{F})$ is a pramart if for every $\varepsilon>0$, there exists $\sigma_{\varepsilon} \in \mathbb{T}$ such that

$$
\forall \sigma, \tau \in \mathbb{T}, \quad \tau \geq \sigma \geq \sigma_{\varepsilon} \Rightarrow P\left(\left\|X_{\sigma}-E^{\mathcal{F}_{\sigma}} X_{\tau}\right\|_{E_{b}^{*}}>\varepsilon\right)<\varepsilon .
$$

Similarly if $\left(X_{n}\right)_{n \in \mathbb{N}}$ is a pramart in $L_{E^{*}}^{1}[E](\mathcal{F})$, then for every $x$ in the unit ball $\bar{B}_{E}$ of $E$, the sequence $\left(\left\langle x, X_{n}\right\rangle\right)_{n \in \mathbb{N}}$ is a pramart in $L_{\mathbb{R}}^{1}(\mathcal{F})$, since we have

$$
\left\|X_{\sigma}-E^{\mathcal{F}_{\sigma}} X_{\tau}\right\|_{E_{b}^{*}}=\sup _{x \in \bar{B}_{E}}\left[\left\langle x, X_{\sigma}-E^{\mathcal{F}_{\sigma}} X_{\tau}\right\rangle\right] .
$$

Here we give an elementary lemma that we will be needed later.

Lemma 5.3. Let $\left(A_{n}\right)_{n \in \mathbb{N}}$ be a sequence in $c w k\left(E_{s}^{*}\right)$ and $D$ denotes a dense sequence in $\bar{B}_{E}$ such that

(i) For every $x \in D, \quad \lim _{n \rightarrow \infty} \delta^{*}\left(x, A_{n}\right)$ exist a.s.

(ii) $\sup _{n \in \mathbb{N}}\left|A_{n}\right|<\infty$.

Then there exists $A_{\infty} \in \operatorname{cwk}\left(E_{s}^{*}\right)$ satisfying

$$
\lim _{n \rightarrow \infty} \delta^{*}\left(x, A_{n}\right)=\delta^{*}\left(x, A_{\infty}\right) \quad(x \in E) .
$$


Proof. For each $x \in D$, define the function $r(\cdot)$ by

$$
r(x)=\lim _{n \rightarrow+\infty} \delta^{*}\left(x, A_{n}\right) .
$$

$r$ is sublinear and continuous because by condition (ii) we have

$$
\begin{aligned}
\sup _{\|x\| \leq 1}|r(x)| & =\sup _{\|x\| \leq 1}\left|\lim _{n \rightarrow \infty} \delta^{*}\left(x, A_{n}\right)\right| \\
& \leq \sup _{n \in \mathbb{N}}\left|A_{n}\right|<\infty .
\end{aligned}
$$

Hence by [17, Lemma 1] there exists $A_{\infty} \in c w k\left(E_{s}^{*}\right)$ such that

$$
r(x)=\delta^{*}\left(x, A_{\infty}\right) \quad \forall x \in \bar{B}_{E} .
$$

Now we are ready to state the decomposition of $c w k\left(E_{s}^{*}\right)$-valued pramarts.

Theorem 5.4. Let $\left(X_{n}\right)_{n \in \mathbb{N}}$ be a bounded pramart in $\mathcal{L}_{c w k\left(E_{s}^{*}\right)}^{1}(\mathcal{F})$. Then there exists $X_{\infty} \in \mathcal{L}_{\text {cwk(E) }}^{1}(\mathcal{F})$ such that

$$
\begin{gathered}
\lim _{n \rightarrow \infty} \delta^{*}\left(x, X_{n}\right)=\delta^{*}\left(x, X_{\infty}\right) \quad \text { a.s. } \quad \forall x \in \bar{B}_{E}, \\
\lim _{n \rightarrow \infty} \mathcal{H}_{E_{b}^{*}}^{*}\left(X_{n}, E^{\mathcal{F}_{n}} X_{\infty}\right)=0 \quad \text { a.s. }
\end{gathered}
$$

Proof. Step 1 Claim: $\lim _{n \rightarrow \infty} \delta^{*}\left(x, X_{n}\right)=\delta^{*}\left(x, X_{\infty}\right)$ a.s. $\forall x \in \bar{B}_{E}$.

Let $D_{1}=\left(e_{j}\right)_{j \in \mathbb{N}}$ denotes a dense sequence in $\bar{B}_{E}$. As $\left(X_{n}\right)_{n \in \mathbb{N}}$ is a bounded pramart in $\mathcal{L}_{c w k\left(E_{s}^{*}\right)}^{1}(\mathcal{F})$, for each $j \in \mathbb{N},\left(\delta^{*}\left(e_{j}, X_{n}\right)\right)_{n \in \mathbb{N}}$ is a bounded real-valued pramart in $L_{\mathbb{R}}^{1}(\mathcal{F})$. So for each $j \in \mathbb{N},\left(\delta^{*}\left(e_{j}, X_{n}\right)\right)_{n \in \mathbb{N}}$ converges a.s. to an integrable function $m_{j}$ in $L_{\mathbb{R}}^{1}(\mathcal{F})$. By hypotheses of theorem, since $\left(X_{n}, \mathcal{F}_{n}\right)_{n \geq 1}$ is a multivalued pramart, then for every $n \in \mathbb{N},\left(\left|X_{n}\right|, \mathcal{F}_{n}\right)_{n \geq 1}$ is a sequence of real subpramarts which are bounded in $L_{\mathbb{R}}^{1}(\mathcal{F})$. So by $[9$, Lemma VIII.2.4.1] we deduce that

$$
\sup _{n \in \mathbb{N}}\left|X_{n}\right|<\infty \quad \text { a.s. }
$$

Hence Lemma 5.3 gives $X_{\infty} \in \operatorname{cwk}\left(E_{s}^{*}\right)$ such that

$$
\lim _{n \rightarrow \infty} \delta^{*}\left(x, X_{n}\right)=\delta^{*}\left(x, X_{\infty}\right) \quad \text { a.s. } \quad \forall x \in \bar{B}_{E} .
$$

Finally by Fatou Lemma, We check that $X_{\infty} \in \mathcal{L}_{c w k\left(E_{s}^{*}\right)}^{1}(\mathcal{F})$.

Step 2 Claim: $\lim _{n \rightarrow \infty} \mathcal{H}_{E_{b}^{*}}^{*}\left(X_{n}, E^{\mathcal{F}_{n}} X_{\infty}\right)=0$ a.s.

Let $D_{1}=\left(e_{j}\right)_{j \in \mathbb{N}}$ denote a dense sequence in $\bar{B}_{E}$. We have

$$
\mathcal{H}_{E_{b}^{*}}^{*}\left(X_{n}, E^{\mathcal{F}_{n}} X_{\infty}\right)=\sup _{j \in \mathbb{N}}\left|\delta^{*}\left(e_{j}, X_{n}\right)-\delta^{*}\left(e_{j}, E^{\mathcal{F}_{n}} X_{\infty}\right)\right| .
$$

As $\left(\delta^{*}\left(e_{j}, X_{n}\right)-\delta^{*}\left(e_{j}, E^{\mathcal{F}_{n}} X_{\infty}\right)\right)_{n \in \mathbb{N}}$ are real-valued pramarts in $L_{\mathbb{R}}^{1}(\mathcal{F})$ which converges a.s. to 0 , and $\left(\left(\left|\delta^{*}\left(e_{j}, X_{n}\right)-\delta^{*}\left(e_{j}, E^{\mathcal{F}_{n}} X_{\infty}\right)\right|\right)_{n \in \mathbb{N}}\right)_{j \in \mathbb{N}}$ is a uniform sequence of positive subramarts, applying Lemma VIII.1.15 in [9] we have

$$
\lim _{n \rightarrow \infty} \mathcal{H}_{E_{b}^{*}}^{*}\left(X_{n}, E^{\mathcal{F}_{n}} X_{\infty}\right)=\lim _{n \rightarrow \infty} \sup _{j \in \mathbb{N}}\left|\delta^{*}\left(e_{j}, X_{n}\right)-\delta^{*}\left(e_{j}, E^{\mathcal{F}_{n}} X_{\infty}\right)\right|
$$




$$
=\sup _{j \in \mathbb{N}} \lim _{n \rightarrow \infty}\left|\delta^{*}\left(e_{j}, X_{n}\right)-\delta^{*}\left(e_{j}, E^{\mathcal{F}_{n}} X_{\infty}\right)\right|=0
$$

almost surely.

Comments: In [4] when the Banach space is weakly compactly generated (WCG), the authors give the weak star Kuratowski ( $w^{*} K$ for short) converges of pramarts in $\mathcal{L}_{c w k\left(E_{s}^{*}\right)}^{1}(\mathcal{F})$. Recall that the Banach space $E$ is weakly compactly generated (WCG) if there exists a weakly compact subset of $E$ whose linear span is dense in $E$.

Corollary 5.5. Let $\left(X_{n}\right)_{n \in \mathbb{N}}$ be a bounded pramart in $L_{E^{*}}^{1}[E](\mathcal{F})$. Then there exist a martingale $\left(Y_{n}\right)_{n \in \mathbb{N}}$ in $L_{E^{*}}^{1}[E](\mathcal{F})$ and a pramart $\left(Z_{n}\right)_{n \in \mathbb{N}}$ in $L_{E^{*}}^{1}[E](\mathcal{F})$ such that $X_{n}=Y_{n}+Z_{n}, \forall n \in \mathbb{N}$ and such that $\left(Z_{n}\right)_{n \in \mathbb{N}}$ norm converges to 0 a.s.

Proof. As $\left(\left\langle x, X_{n}\right\rangle\right)_{n \in \mathbb{N}}$ is a real-valued bounded pramart in $L_{\mathbb{R}}^{1}$ for each $x \in$ $\bar{B}_{E},\left(\left\langle x, X_{n}\right\rangle\right)_{n \in \mathbb{N}}$ converges a.s to an integrable function $m_{x}$. Then Theorem 5.4 (ii) provides a $X_{\infty} \in L_{E^{*}}^{1}[E](\mathcal{F})$ such that

$$
\lim _{n \rightarrow \infty}\left\|X_{n}-E^{\mathcal{F}_{n}} X_{\infty}\right\|_{E_{b}^{*}}=0 \text { a.s. }
$$

the result follows by putting $Y_{n}=E^{\mathcal{F}_{n}} X_{\infty}$ and $Z_{n}=X_{n}-E^{\mathcal{F}_{n}} X_{\infty}$.

Now we state the existence of martingales selectors for $c w k\left(E_{s}^{*}\right)$-valued supermartingales $\left(X_{n}\right)_{n \geq 1}$ in $\mathcal{L}_{c w k\left(E_{s}^{*}\right)}^{1}(\mathcal{F})$ via a projective limit technique. See ([11, Proposition 3.7]) for details. For this purpose we shall recall the definition of the projective limit of a sequence of sets. Let $\left(\Gamma_{n}\right)_{n \geq 1}$ be a sequence of sets and for any $m, n \geq 1$ such that $m \leq n$, a map $u_{m n}: \Gamma_{n} \longrightarrow \Gamma_{m}$. Also assume the two following hypotheses:

(i) $\forall m \geq 1, u_{m m}=i d_{\Gamma_{m}}=$ the identity map of $\Gamma_{m}$.

(ii) $\forall m, n, p \geq 1$ such that $m \leq n \leq p, u_{m p}=u_{m n} \circ u_{n p}$.

The sequence $\left(\Gamma_{n}\right)_{n \geq 1}$, together with the maps $u_{m n}$ is called a projective system. If the $\Gamma_{n}$ are topological spaces and if the $u_{m n}$ are continuous we speak of a projective system of topological spaces. Let $\Gamma$ be the cartesian product of the $\Gamma_{n}$ for $n \geq 1$ and $p r_{n}$ the projection from $\Gamma$ onto $\Gamma_{n}$. The subset $S$ of $\Gamma$ defined by

$$
S:=\left\{x=\left(x_{n}\right)_{n \geq 1} / p r_{m}(x)=u_{m n} \circ p r_{n}(x) \quad \forall m, n \geq 1, m \leq n\right\}
$$

is called the projective limit of the projective system defined above.

Lemma 5.6. Let $\left(X_{n}, \mathcal{F}_{n}\right)_{n \geq 1}$ be a supermartingales in $\mathcal{L}_{c w k\left(E_{s}^{*}\right)}^{1}(\mathcal{F})$. Then $\left(X_{n}, \mathcal{F}_{n}\right)_{n \geq 1}$ admits a martingale selector $\left(f_{n}, \mathcal{F}_{n}\right)_{n \geq 1}$ in $L_{E^{*}}^{1}[E](\mathcal{F})$.

Proof. By [6, Theorem VIII.34], for $m<n$ and for $f \in L_{E^{*}}^{1}[E]\left(\mathcal{F}_{n}\right)$, the conditional expectation $E^{\mathcal{F}_{m}} f$ exists and belongs to the space $L_{E^{*}}^{1}[E]\left(\mathcal{F}_{m}\right)$. Now thinks to Proposition VIII.33 in [6]

$$
E^{\mathcal{F}_{m}}: L_{E^{*}}^{1}[E]\left(\mathcal{F}_{n}\right) \longrightarrow L_{E^{*}}^{1}[E]\left(\mathcal{F}_{m}\right)
$$


is continuous for the topologies

$$
\sigma\left(L_{E^{*}}^{1}[E]\left(\mathcal{F}_{n}\right), L_{E}^{\infty}\left(\mathcal{F}_{n}\right)\right)
$$

and

$$
\sigma\left(L_{E^{*}}^{1}[E]\left(\mathcal{F}_{m}\right), L_{E}^{\infty}\left(\mathcal{F}_{m}\right)\right)
$$

respectively. Further by $\left[6\right.$, Theorem VIII.34] the $L_{E^{*}}^{1}[E]\left(\mathcal{F}_{n}\right)$-selectors set $S_{X_{n}}^{1}\left(\mathcal{F}_{n}\right)$ is convex $\sigma\left(L_{E^{*}}^{1}[E]\left(\mathcal{F}_{n}\right), L_{E}^{\infty}\left(\mathcal{F}_{n}\right)\right)$ compact. Now let us set

$$
u_{m n}(f):=E^{\mathcal{F}_{m}} f, \quad \forall f \in S_{X_{n}}^{1}\left(\mathcal{F}_{n}\right) .
$$

Then by the supermartingale property we have

$$
u_{m n}\left(S_{X_{n}}^{1}\left(\mathcal{F}_{n}\right)\right) \subset\left(S_{X_{m}}^{1}\left(\mathcal{F}_{m}\right)\right) .
$$

Therefore the sequence $\left(S_{X_{n}}^{1}\left(\mathcal{F}_{n}\right)\right)_{n \geq 1}$ together with the sequence of continuous mappings $\left(u_{m n}\right)_{m<n}$ is a projective system of compact spaces in $L_{E^{*}}^{1}[E](\mathcal{F})$. Therefore, by [2, Proposition 8, p.I.64] this system admits a nonempty projective limit which is the set $M S\left(X_{n}\right)$ of $L_{E^{*}}^{1}[E](\mathcal{F})$-martingales selectors of $\left(X_{n}\right)$. (i.e., any member $\left(f_{k}\right)_{k \geq 1}$ of the projective limit satisfies, for any $m, n \in \mathbb{N}$ such that $m<n$,

$$
p r_{m}\left(\left(f_{k}\right)\right)=u_{m n} \circ p r_{n}\left(\left(f_{k}\right)\right)
$$

or, equivalently, $f_{m}=E^{\mathcal{F}_{m}} f_{n}$. So we have $\left.\left(f_{k}\right) \in M S\left(X_{n}\right)\right)$.

Now we proceed to the existence of pramart selector of pramart in $\mathcal{L}_{c w k\left(E_{s}^{*}\right)}^{1}(\mathcal{F})$.

Theorem 5.7. Let $\left(X_{n}\right)_{n \in \mathbb{N}}$ be a bounded pramart in $\mathcal{L}_{c w k\left(E_{s}^{*}\right)}^{1}(\mathcal{F})$, if assumption $(A)$ holds. Then $\left(X_{n}\right)_{n \in \mathbb{N}}$ admits a pramart selector $\left(f_{n}\right)_{n \geq 1}$ in $L_{E^{*}}^{1}[E](\mathcal{F})$, that is $\left(f_{n}\right)_{n \in \mathbb{N}}$ is an integrable pramart and $f_{n}(\omega) \in X_{n}(\omega)$ for all $n \geq 1$ and for all $\omega \in \Omega$.

Proof. Applying Theorem 5.4 to $\left(X_{n}\right)_{n \geq 1}$ which provides a multifunction $X_{\infty} \in$ $\mathcal{L}_{c w k\left(E_{s}^{*}\right)}^{1}(\mathcal{F})$ such that

$$
\lim _{n \rightarrow \infty} \mathcal{H}_{E_{b}^{*}}^{*}\left(X_{n}, E^{\mathcal{F}_{n}} X_{\infty}\right)=0 \text { a.s. }
$$

let us set $M_{n}=E^{\mathcal{F}_{n}} X_{\infty}$ and $r_{n}=\mathcal{H}_{E_{b}^{*}}^{*}\left(X_{n}, E^{\mathcal{F}_{n}} X_{\infty}\right)+\frac{1}{2^{n}}$. Since $\left(M_{n}\right)_{n \geq 1}$ is a $c w k\left(E_{s}^{*}\right)$-valued martingale, by Lemma 5.6 there exists $\left(g_{n}\right)_{n \geq 1}$ a martingale selector of $M_{n}$. By same argument in the proof of Theorem 4.6, for each $g_{n}$ we pick a $\mathcal{F}_{n}$-measurable selector of $X_{n}$ such that

$$
\left\|f_{n}-g_{n}\right\|_{E_{b}^{*}} \leq r_{n} .
$$

Writing $f_{n}=g_{n}+\left(f_{n}-g_{n}\right)=g_{n}+z_{n}$ where $z_{n}=f_{n}-g_{n}$, by $(5.7 .1)\left(z_{n}\right)_{n \geq 1}$ converge a.s to zero when $n$ goes to $\infty$, and for all $\tau \in \mathbb{T}$ we have

$$
\int_{\Omega}\left\|z_{\tau}\right\| d P \leq \int_{\Omega}\left\|f_{\tau}\right\| d P+\int_{\Omega}\left|X_{\infty}\right| d P<\infty
$$




$$
\sup _{\tau \in \mathbb{T}} \int_{\Omega}\left\|z_{\tau}\right\| d P \leq \sup _{\tau \in \mathbb{T}} \int_{\Omega}\left\|f_{\tau}\right\| d P+\int_{\Omega}\left|X_{\infty}\right| d P<\infty,
$$

then

$$
\sup _{\tau \in \mathbb{T}} \int_{\Omega}\left\|z_{\tau}\right\| d P<\infty
$$

Hence by Lemma $4.2, z_{n}$ is a pramart. By Corollary $5.5\left(f_{n}\right)_{n \geq 1}$ is a pramart selector, because $\left(g_{n}\right)_{n \geq 1}$ is a $L_{E^{*}}^{1}[E](\mathcal{F})$ martingale and $\left(z_{n}\right)_{n \geq 1}$ is a pramart norm converges to 0 a.s.

Acknowledgments. We thank Professor C. Castaing for his useful discussions and valuable comments.

\section{References}

[1] F. Akhiat, C. Castaing and F. Ezzaki, Some various convergence results for multivalued martingales, Adv. Math. Econ. 13 (2010), 1-33.

[2] N. Bourbaki, Elèments de mathematiques, Topologie Génerale, Chaps. 1-4, Hermann, Paris. 1971

[3] C. Castaing, F. Ezzaki, M. Lavie, and M. Saadoune, Weak star convergence of martingales in a dual space, Function spaces IX, 45-73, Banach Center Publ., 92, Polish Acad. Sci. Inst. Math., Warsaw, 2011.

[4] C. Castaing, F. Ezzaki, and K. Tahri, Convergence of multivalued pramarts, J. Nonlinear Convex Anal. 11 (2010), no. 2, 243-266.

[5] C. Castaing and M. Saadoune, Convergences in a dual space with applications to Fatou lemma, Adv. Math. Econ. 12 (2009), 23-69.

[6] C. Castaing and M. Valadier, Convex Analysis and Measurable Multifunctions, Lectures Notes, 580, Springer-Verlag, Berlin 1977.

[7] A. Choukairi-Dini, M-convergence, et régularité des martingales multivoques: epimartingales, J. Multivariate Anal. 33 (1990), no. 1, 49-71.

[8] _ On almost sure convergence of vector valued pramarts and multivalued pramarts, J. Convex Anal. 3 (1996), no. 2, 245-254.

[9] L. Egghe, Stopping time techniques for analysts and probabilists, Cambridge Univ. Press, London and New York, 1984.

[10] Strong convergence of pramarts in Banach spaces, Canad. J. Math. 33 (1981), no. 2, 357-361.

[11] C. Hess, On multivalued martingales whose values may be unbounded: martingale selectors and Mosco convergence, J. Multivariate Anal. 39 (1991), no. 1, 175-201.

[12] F. Hiai and H. Umegaki, Integrals, conditional expectations and martingales of multivalued functions, J. Multivariate Anal. 7 (1977), no. 1, 149-182.

[13] S. Li and Y. Ogura, Convergnece of set valued sub-and supermartingales in the Kuratowski-Mosco sense, Ann. Prob. 26 (1998), no. 3, 1384-1402.

[14] D. Q. Lu'u, Representations and regularity of multivalued martingales, Acta Math. Vietnam. 6 (1981), no. 2, 29-40.

[15] _ On convergence of multivalued asymptotic martingales, Séminaire d'analyse convexe, Vol. 14 (Montpellier, 1984), Exp. No. 5, 23 pp., Univ. Sci. Tech. Languedoc, Montpellier, 1984.

[16] - Representations of multivalued (regular) uniform amarts, Séminaire d'analyse convexe, Vol. 14 (Montpellier, 1984), Exp. No. 9, 33 pp., Univ. Sci. Tech. Languedoc, Montpellier, 1984.

[17] J. Neveu, Convergence presque sûr de martingales multivoques, Ann. Inst. H. Poincaré Sect. B (N.S.) 8 (1972), no. 1, 1-7. 
[18] M. Valadier, On conditional expectation of random sets, Ann. Mat. Pura Appl. (4) 126 (1980), 81-91.

[19] Z. P. Wang and X. H. Xue, On convergence and closedness of multivalued martingales, Trans. Amer. Math. Soc. 341 (1994), no. 2, 807-827.

[20] _ Some remarks on pramarts and mils, Glasg. Math. J. 35 (1993), no. 2, 239-251.

FetTah Akhiat

Laboratoire Modélisation et Calcul Scientifique

Département de Mathématiques, Faculté des Sciences et Techniques BP 2202, FÈs MAROC

E-mail address: akhiatfettah@yahoo.fr

FATIMA EZZAKI

Laboratoire Modélisation et Calcul Scientifique

Département de Mathématiques, Faculté des Sciences et Techniques

BP 2202, FÈs Maroc

E-mail address: fatimaezzaki@yahoo.fr 